ERIQUES GONÇALVES DA SILVA

\title{
AVALIAÇÃO DA CRIPTOCOCOSE EXPERIMENTAL SISTÊMICA EM CAMUNDONGOS BALB/C E TERAPÊUTICA COM ANFOTERICINA B, FLUCONAZOL E ASSOCIAÇÃO.
}

Tese apresentada ao Instituto de Ciências Biomédicas da Universidade de São Paulo, para obtenção do titulo de Doutor em Ciências.

Área de Concentração: Microbiologia

Orientadora:Profa.Dra .Claudete Rodrigues Paula

São Paulo 


\section{RESUMO}

SILVA, E.G. Avaliação da criptococose experimental sistêmica em camundongos BALB/c e terapêutica com anfotericina B, fluconazol e associação. 2007. 100 f. Tese (Doutorado em Ciências) - Instituto de Ciências Biomédicas, Universidade de São Paulo, 2007.**

A criptococose clinica foi observada por volta do primeiro dia da inoculação e a sobrevida dos animais 15 dias após-inoculação $(\mathrm{PI})$. Isolamos $C$. neoformans no cérebro a partir do $5^{\circ}$ dia $(\mathrm{PI})$ e no pulmão a partir $11^{\circ}$ dia $(\mathrm{PI})$. No $1^{\circ}$ dia $(\mathrm{PI})$ observamos por meio do tecido cerebral que já havia um quadro inicial de infecção, presença de edema, que evoluiu durante todo o período. C. neoformans foi visualizado primeiramente nos vasos capilares, o que nos leva a sugerir que seja esta rota importante para a entrada da levedura no órgão. A meningite aguda aconteceu por volta do $7^{\circ}$ dia quando visualizamos o microrganismo na meninge e com um discreto infiltrado inflamatório. A partir do $13^{\circ}$ dia a doença evoluiu para crônica permanecendo até o óbito dos animais. A monoterapia com anfotericina $B$ (AMB) reduziu a sobrevida dos animais, enquanto que, o tratamento isolado com fluconazol (FLC) prolongou a mesma. A associação AMB-FLC foi eficaz, quando iniciamos o tratamento 24 horas $(\mathrm{PI})$, enquanto que, o mesmo tratamento iniciado a partir do $7^{\circ}$ dia quando já tínhamos um quadro compatível de meningite aguda não foi satisfatório. É importante ressaltar que os resultados descritos foram referentes à inoculação com isolado sensível in vitro ao fluconazol, enquanto que, o tratamento não resultou em êxito quando empregamos isolado resistente in vitro ao fluconazol.

Palavras-chave: Criptococose experimental. Associação terapêutica. Anfotericina B. Fluconazol. Cryptococcus neoformans. Monoterapia. 


\begin{abstract}
SILVA, E.G. Evaluation of experimental systemic cryptococcosis in BALB/c mice, and its treatment with amphotericin B and fluconazole, alone and in association. 2007. $100 \mathrm{f}$. Master thesis (Sciences) - Instituto de Ciências Biomédicas, Universidade de São Paulo, (2007). **
\end{abstract}

Clinical cryptococcosis was observed on day 1 postinoculation $(\mathrm{PI})$, and the animals survived until day $15 \mathrm{PI}$. C. neoformans was isolated from brain tissue starting on day $5 \mathrm{PI}$, and from lung tissue starting on day $11 \mathrm{PI}$. On day $1 \mathrm{PI}$, signs of infection were already observed in brain tissue, with presence of edema, which evolved throughout the period. C. neoformans was first seen in the capillaries, suggesting that this is an important route for the entrance of the yeast into this organ. Acute meningitis occurred around day $7 \mathrm{Pl}$, when the microorganism was observed in the meninges, along with a discrete inflammatory infiltrate. From day $13 \mathrm{PI}$ onward the disease became chronic, persisting until the death of the animals. Treatment with amphotericin B (AMB) alone shortened the animals' survival, while treatment with fluconazole (FLC) alone lengthened it. Treatment with the two drugs in association was effective when treatment was begun at 24 hours PI, however, when treatment was begun at day $7 \mathrm{PI}$, already with signs of acute meningitis, the effectiveness was unsatisfactory. It is important to emphasize that these results relate to inoculation with an isolate susceptible in vitro to fluconazole, while the treatment was not effective for an isolate resistant in vitro to fluconazole.

Key words: Experimental cryptococcosis. Therapêutic association. Amphotericin B. Fluconazole. Cryptococcus neoformans. Monotherapy. 


\section{INTRODUÇÃO}

\subsection{Cryptococcus neoformans}

Cryptococcus neoformans variedade neoformans é uma levedura patogênica oportunista encontrada em ocos de arvores (REIMÃO et al., 2007) e excretas de pombos e outros pássaros (MONTENEGRO e PAULA, 2000; LACAZ et al., 2002; LITVINTSEVA et al., 2005; CHEE e LEE, 2005; BARONI et al., 2006; CAFARCHIA et al., 2006).

Estas excretas constituem fator importante na transmissão de $C$. neoformans em área urbana (SOARES et al., 2005), bem como em zona rural (DELGADO et al., 2005).

É o agente etiológico da criptococose, que atinge pacientes imunodeprimidos (HIV-positivos) e imunossuprimidos por terapias ou outras doenças (CASADEVALL e PERFECT, 1998), apresentando um tropismo pelo sistema nervoso central que é fator determinante do óbito em infecção experimental (CASADEVALL e PERFECT, 1998; SILVA et al., 2006).

Duas variedades são conhecidas: var. gattii (sorotipos B e C) e var. neoformans (sorotipos A, D e AD), sendo que FRANZOT et al., 1999, propôs subdividir a variedade neoformans em, var. neoformans (sorotipo D) e var. grubii (sorotipo A). No Brasil, o sorotipo A, Cryptococcus neoformans var. grubii, é o mais prevalente (CASALI et al., 2003; FERNANDES et al., 2003; KOBAYASHI et al., 2005). Estudo em diferentes locais de Seul na Coréia com amostras isoladas de excretas de pombos revelou que todas as amostras pertenciam ao sorotipo A (CHEE e LEE, 2005). Na Colômbia pesquisas realizadas com amostras isoladas de excretas de pombos revelaram que $31 \%$ pertenciam ao sorotipo A (QUINTERO et a.I, 2005). $\mathrm{Na}$ Espanha o sorotipo prevalente de isolados ambientais é o $D$ e o $A D$, enquanto 
que, em isolados clínicos o sorotipo A é o mais freqüente (BARÓ et al., 1999).

Pesquisas realizadas com amostras isoladas de pacientes de 40 localidades de Nova lorque mostraram que, $85 \%$ dos isolados pertenciam ao sorotipo D, indicando uma significativa diferença quando comparados com estudos anteriores, que demonstravam a prevalência do sorotipo A em outros isolados norte americanos. (STEENBERGEN e CASADEVALL, 2000).

\subsection{CRIPTOCOCOSE}

É uma micose sistêmica subaguda ou crônica, de natureza cosmopolita, responsável por uma alta taxa de mortalidade e morbidade especialmente em pacientes com AIDS (FERNANDES et al., 2000; MOREIRA et al., 2006); é considerada a quarta infecção mais comum em individuos imunocompromissados (KOVACS et al., 1985) podendo ser fatal quando não tratada (CASADEVAL e PERFECT, 1998).

A maioria das infecções por C. neoformans são causadas pelo sorotipo A (CASADEVALL e PERFECT, 1998; CASALI et al., 2003). Na América Latina, pesquisa com isolados de pacientes com AIDS revelou que 96,8\% pertenciam ao sorotipo A (CALVO et al., 2001). A prevalência do sorotipo A em isolados clínicos de pacientes com criptococose pode ser devido à elevada temperatura corpórea causada pela febre (MARTINEZ et al., 2001).

Cryptococcus neoformans pode ser disseminado pelo ar e infectar humanos, usando como porta de entrada as vias respiratórias, alojando-se primariamente nos pulmões (BUCHANAN e MURPHY, 1998; LACAZ et al., 2002). Há evidências consideráveis que ocorre uma fase de latência da levedura após a 
sua inalação (GARCIA-HERMOSO et al., 1999). A doença se desenvolve como resultado da disseminação hematogenica da levedura indo do pulmão para o cérebro (CHANG et al., 2004a). Para causar a meningoencefalite as células do fungo tem que sobreviver na circulação sangüínea e atravessar a barreira hematoencefálica (BBB) (RUBIN, 1999), provavelmente alcançando o sistema nervoso central pelo endotélio microvascular do cérebro (KIM, 2003).

A patogenicidade de $C$. neoformans varia entre os isolados e ainda não é completamente explicada (PAL, 2005).

A doença deve ser considerada realizando-se diagnóstico diferencial das infecções oportunistas nos indivíduos imunodeprimidos, sendo fundamental o diagnóstico precoce para reduzir a morbidade e mortalidade desta micose que na maioria dos casos se apresenta na forma de meningoencefalite (FILIU et al., 2002; PAPPALARDO e MELHEM, 2003) podendo ser considerado como marcador de sentinela na infecção pelo HIV (LEITE et al., 2004; LIZARAZO et al., 2007; AKCAGLAR et al., 2007). Estima-se que de 25 a $30 \%$ de pacientes com AIDS morrerão em conseqüência da doença (ROBINSON et al., 1999; FORD, 2000; PITISUTTITHUM et al., 2001).

A incidência da infecção por Cryptococcus neoformans tem aumentado muito desde a década de 70 , sendo que nos anos 90 , em conseqüência da epidemia de AIDS, a criptococose tem aumentado de modo significativo (MITCHELL e PERFECT, 1995; CASADEVALL e PERFECT, 1998). A infecção pelo vírus HIV continua sendo um importante fator para o desenvolvimento da criptococose no sistema nervoso central (LAKSHMI et al., 2007). 


\subsection{SUSCETIBILIDADE AOS ANTIFUNGICOS}

A avaliação da suscetibilidade de C. neoformans aos antifúngicos é de grande interesse considerando a alta freqüência e as severas manifestações clinicas da infecção por esta levedura (BARCHIESI et al., 1995).

Os testes de susceptibilidade antifúngica para leveduras ainda requerem mais estudos, devido às divergências quanto ao uso de drogas, a diversidade dos organismos e as metodologias especificas. Existem alguns métodos, como os que empregam discos de papel de filtro impregnados em solução do antifúngico; os métodos de macrodiluição e microdiluição em caldo proposto pelo NCCLS ("National Committee for Clinical Laboratory Standards"), atualmente denominado "CLSI", ("Clinical and Laboratory Standards Institute") e o método EUCAST ("European Committee on Antimicrobial Susceptibility Testing") (RODRIGUESTUDELA, 2002).

Alem da recomendação do CLSI no uso do meio RPMI 1640 + MOPS acrescido de $2 \%$ de glicose, o meio YNB ("Yeast Nitrogen Base") pH 7.0 também é um excelente meio na execução dos testes de susceptibilidade aos antifúngicos através da técnica do E-test (PETROU e SHANSON, 2000).

Rodrigues-Tudela et al. (2000), estudaram três meios (RPMI 1640, RPMI 1640-2\% glicose e (YNB) indicado pelo (CLSI) e verificaram a habilidade de crescimento e a concentração inibitória mínima de Cryptococcus neoformans em microplacas incubadas a $35^{\circ} \mathrm{C}$ por 48 horas. Os resultados obtidos mostraram que no meio RPMI 1640 o crescimento foi ótimo, e neste meio suplementado com $2 \%$ de glicose o crescimento não melhorou. Comparando os CIMs para anfotericina B, entre estes meios, observaram ser muito pequena a diferença entre susceptibilidade e 
resistência da levedura. No meio YNB, obtiveram resultados de MICs melhores para fluorocitosina e fluconazol.

Montenegro e Paula (2000) em seu estudo com cepas ambientais de São Paulo, assim como Baroni (2001) estudando cepas ambientais do Rio de Janeiro e utilizaram o E-test com o meio YNB modificado.

O método de difusão em disco usando ágar Müeller - Hinton acrescido de glicose e azul de metileno tem uma aproximação útil para determinar a susceptibilidade de C. neoformans ao fluconazol (PAFALLER et al., 2004).

Estudo realizado por Dias et al., (2006) revelou que exceto a anfotericina B a correlação entre o E-test e o método EUCAST é muito boa.

\subsubsection{METODO DO E-TEST}

O E-test é um método potencial para testes de susceptibilidade aos antifúngicos produzidos pela AB BIODISK (Solna, Suécia). Ele é composto de fita graduada com valores expressos em $\mu \mathrm{g} / \mathrm{mL}$ contendo um gradiente de concentração de uma droga pré-definida. Neste método é possível se obter uma reprodutibilidade quantitativa, nas leituras das concentrações inibitórias mínimas das drogas. É um excelente método para se discriminar cepas sensíveis e resistentes de C. neoformans (LOZANO-CHIU et al., 1998).

Com exceção do itraconazol, a correlação do método de E-test e o método de microdiluição (CLSI) é muito boa. O método é simples, e os resultados podem ser lidos entre 24 e 48 horas após a inoculação (CHANG et al, 2001). O E-

test é uma excelente alternativa no laboratório clinico para se determinar à susceptibilidade ao fluconazol e fluorocitosina (ALLER et al, 2000a). É muito útil para 
se determinar à susceptibilidade de $C$. neoformans in vitro aos antifúngicos para anfotericina B (MAXWELL et al., 2003). Exceto ao itraconazol, a correlação do método E-test e o método de microdiluição (CLSI) é muito boa (MAXWELL et al., 2003) e fidedigna com relação ao fluconazol (MATAR et al., 2003).

\subsubsection{SUSCEPTIBILIDADE IN VITRO}

Estudo realizado por Alves et al., (2001) demonstrou que as concentrações inibitórias mínimas (CIMs) entre isolados de pacientes HIV-positivos e isolados ambientais foram semelhantes para anfotericina $B$, enquanto que, frente ao fluconazol os isolados clínicos mostraram ser menos sensíveis do que os provenientes do ambiente. A susceptibilidade entre isolados ambientais antes e após a inoculação em camundongos BALB/c foram semelhantes frente ao fluconazol, (SILVA, 2003). Cepas ambientais e clinicas mostraram semelhança com relação ao perfil de susceptibilidade, quanto a anfotericina B e ao fluconazol (SOUZA et al., 2005). Pesquisa in vitro com cepas isoladas de excretas de pombos apresentaram $100 \%$ de sensibilidade a anfotericina B e ao fluconazol (KOBAYASHI et al., 2005). A criptococose em humanos pode ser adquirida a partir de cepas ambientais com CIM semelhante às cepas clinicas para agentes antifúngicos (DELGADO et al., 2005). Isolados do ambiente podem mostrar valores de CIMs altos in vitro sugerindo que estes isolados possam ter resistência primária a algumas drogas (PEDROSO et al., 2006).

No período de 2003, estudo com isolados clínicos de C. neoformans em Taiwan revelou uma diminuição da sensibilidade a anfotericina B e ao fluconazol, (HSUEH et al., 2005). 
A susceptibilidade a antifúngicos in vitro usando as técnicas atuais pode não predizer antecipadamente bons resultados clínicos em pacientes com criptococose (DANNAOUI et al., 2006).

\subsection{TRATAMENTO}

O fato das infecções fungicas representarem parasitismo de um organismo eucariótico sobre outro eucariótico, com diferenças fisiológicas muito pequenas, é necessário que as drogas antifúngicas tenham aplicação clinica adequada, com o mínimo de efeitos colaterais (LACAZ e NEGRO, 1991).

Um número limitado de agentes antifúngicos inclusive azólicos, poliênicos, análogos da pirimidina são usados hoje para combater infecções causadas por leveduras patogênicas (LACAZ et al., 2002).

Enquanto que fatores clínicos podem contribuir para o insucesso no tratamento destas doenças, a exposição do cryptococcus aos agentes antifúngicos pode limitar a ação dos mesmos por serem intrinsicamente resistentes ou por adquirirem resistência especifica (SANGLARD, 2002).

O tratamento da maioria das infecções estão embasadas nos resultados da sensibilidade in vitro, esses resultados podem predizer a resposta clinica, porem, esta resposta depende de vários outros fatores intrínsecos ao antifúngico bem como da interação patógeno versus hospedeiro. Devido a essa multiplicidade de fatores é evidente que existam discrepâncias entre os resultados de sensibilidade in vitro e in vivo (RIVAS e SERRANO, 2003). 
Segundo Pfaller et al., (1999) e Brandt et al., (2001), o fracasso no tratamento geralmente não é devido à resistência adquirida a droga e sim pela resistência inicial da cepa ao antifúngico.

O uso crescente de agentes antifúngicos contra leveduras patogênicas em pacientes imunocomprometidos tem feito com que cepas resistentes a estes agentes sejam selecionadas, sendo necessária uma maior vigilância quanto ao uso indiscriminado destas drogas (PFALLER et al., 2005).

O regime inicial no tratamento da criptococose continua sendo com anfotericina B com ou sem fluorocitosina, enquanto que, para tratamentos mais longos a droga de escolha é o fluconazol (SAAG et al., 2000).

\subsubsection{ANFOTERICINA B}

A anfotericina B é um antibiótico poliênico macrociclico produzido pelo actinomiceto Streptomyces nodosus com atividade fungicida contra inúmeros fungos, é comumente usada no tratamento de infecções sistêmicas causadas por leveduras em pacientes imunocomprometidos (BODEY, 1986; WASAN et al., 1997). O efeito fungicida da droga contra C. neoformans depende da concentração administrada (KLEPSER et al., 1998). A ação da anfotericina B depende da concentração usada, do tipo celular e de um bom veiculo para a droga. (ARAUJO et al., 2005).

Este fármaco age, principalmente, ligando-se ao esterol da membrana da levedura, especialmente no ergosterol, danificando esta estrutura, produzindo poros. Esta ligação altera a permeabilidade da membrana, causando vazamento de cátions e íons conduzindo, eventualmente, a morte celular (SUGAR, 1995a), interage diretamente com o ergosterol da membrana celular fúngica, não interferindo na 
síntese da membrana do fungo, somente a desestabiliza (THIPINE et al., 1999). ergosterol é necessário na manutenção da permeabilidade e fluidez da membrana (KELLY et al., 1990), garantindo a modulação de enzimas ligadas à membrana plasmática (AOKI et al., 1993). A ausência do ergosterol e o acumulo de seus precursores afetam a estrutura da membrana plasmática e a absorção de vários nutrientes, tornando os fungos vulneráveis a danos (GEORPAPADOKOU e WALSH, 1994, 1996).

Anfotericina B pode afetar a morfologia da cápsula polissacaridica de $C$. neoformans sugerindo um mecanismo de ação adicional na eficácia da criptococose (ZARAGOZA et al., 2005).

Por mais de 30 anos anfotericina B tem sido uma das mais importantes drogas usadas em infecções fungicas severas. Porém, o uso tem sido limitado devido aos vários efeitos adversos sendo o mais importante sua nefrotoxicidade (DERAY, 2002; MEDRANO et al., 2005). Tanto na formulação convencional como na lipídica sua eficácia se equivale (CHEN, 2002).

Por ter pouca absorção no trato gastrointestinal, em infecções sistêmicas a droga deve ser administrada por via intravenosa. Em altas concentrações alcançam o fígado, baço, pulmão e rins. Sua penetração no liquido celafoloraquidiano (LCR) é muito baixa (2 a 4\%). Sua eliminação é lenta fundamentalmente por via biliar $(<15 \%)$ e renal $(3 \%)$. Em alguns tecidos como o fígado, a anfotericina B pode ser detectada ate 12 meses após a sua administração.

Na urina e no soro a droga pode ser detectada até sete semanas após um curso de terapia. A droga entra nos líquidos pleural, peritonial e sinovial atingindo cerca de $50 \%$ das concentrações séricas. O liquor apresenta concentrações de um terço à metade das do soro. No corpo humano, a maior parte da anfotericina B 
provavelmente está ligada à membrana que contêm colesterol em locais tissulares (NEU, 1994). A principal via para a disposição da droga não é conhecida, parte é excretada pelas vias biliares e apenas 3\% de uma dose é eliminada na urina com concentrações equivalentes às do plasma (NEU, 1994).

\subsubsection{FLUCONAZOL}

Antifúngico triazólico, que age na inibição da síntese de esteroides fungicos e praticamente não altera a síntese de colesterol dos mamíferos, sendo menos tóxico e melhor absorvido que os outros azóis (WALSH et al., 1997).

É facilmente solúvel em água, sua absorção por via oral é completa e muito rápida. Uma das características mais importantes da droga é a sua elevada penetração nos líquidos biológicos de todos os organismos, no liquido cefalorraquidiano de pacientes com meningite, alcançando níveis de $90 \%$.

O principal mecanismo de ação dos azólicos é a inibição da biossíntese do ergosterol, que é importante para a integridade e a manutenção da função da membrana celular dos fungos. Os imidazóis inibem a incorporação do acetato de ergosterol, inibindo a lanosterol dimetilase, por interferência no citocromo P-450 da levedura, trazendo como conseqüência alterações na fluidez e permeabilidade da membrana citoplasmática do fungo, prejudicando a captação dos nutrientes, o que se traduz por inibição do crescimento fungico, originando alterações morfológicas que resultam em necrose celular (RICHARDSON e WARNOCK, 1993). A monoterapia com azois só é eficaz na criptococose primaria pulmonar (JUSTNUBLING, 1994). Pesquisa realizada in vitro revelou que independente da concentração do fluconazol esta droga tem efeito fungistático contra $C$. neoformans 
(KLEPSER et al., 1998), enquanto que, em modelo animal com criptococose moderada, o fluconazol administrado em altas doses tem atividade fungicida (DING et al., 1997). A terapia com fluconazol pode ser efetiva em pacientes imunocompetentes com criptococose pulmonar (NÚÑEZ et al., 2000). É uma excelente droga quando usada na prevenção e tratamento de infecção por $C$. neoformans (LUMBRERAS et al., 2003).

A administração de altas doses de fluconazol como profilaxia ou pósinfecção por C. neoformans pode ser altamente eficaz no tratamento e na prevenção de meningoencefalites (HOSSAIN et al., 2002). A profilaxia primária com fluconazol é efetiva reduzindo a incidência da criptococose em adultos HIV-positvos em estado avançado (CHANG et al., 2005) e pode ser eficaz na prevenção da criptococose invasiva (CANTEY et al., 2005).

A monoterapia com fluconazol pode ser uma opção no tratamento de pacientes com criptococose associada a AIDS em paises com poucos recursos e acesso limitado a terapias antifúngicas (SCHAARS et al., 2006).

A criptococose em pacientes com AIDS raramente é curada, e o fluconazol é a droga de escolha necessária para a manutenção do tratamento (POWDERLY et al., 1992, 2000). O uso indiscriminado deste fármaco em pacientes com AIDS, a longo prazo, pode ainda resultar em resistência (BRANDT et al., 2001; SANAT et al., 2006; ROGERS, 2006). Estudo com cepas clinicas homogêneas demonstrou a ocorrência de heteroresistencia ao fluconazol, segundo esse mesmo estudo isso ocorreu devido a seleção de clones de heteroresistencia e a indução através da exposição ao fluconazol (YAMAZUMI et al., 2003).

O aparecimento de cepas resistentes à droga intensificou a necessidade da avaliação in vitro da susceptibilidade de C. neoformans ao fluconazol (ESPINEL- 
INGROFF, 1997). A susceptibilidade ao fluconazol in vitro pode predizer resposta confiante in vivo (LARSEN et al., 2005). O estudo da CIM in vitro de isolados de pacientes durante a terapia com a droga, como também de pacientes em que a terapia tenha falhado, pode predizer uma potencial resposta clinica a terapia com o antifúngico e ajudar a identificar pacientes que não responderão a terapia com fluconazol (ALLER et al., 2000b).

Como a epidemia da AIDS cresce juntamente com a exposição adicional as drogas azolicas, é muito importante e previdente que seja acompanhada a incidência de resistência de isolados de c. neoformans a este grupo de antifúngicos (RIBEIRO et al., 2005).

Pesquisa quanto à susceptibilidade in vitro realizado por Aller et al., (2007) na Espanha revelou que nos últimos 11 anos a resistência ao fluconazol tem diminuído.

\subsection{ASSOCIAÇÃO TERAPEUTICA}

A associação terapêutica é uma promissora forma usada para aumentar a porcentagem de sucesso no tratamento, porem, se faz necessário mais estudos clínicos controlados para verificar a eficácia destas associações (SHAO et al., 2006). Fármacos associados no tratamento da criptococose tem sido estudados por muito tempo no intuito de descobrir qual a melhor associação, porem, estes estudos tem sido realizados in vitro e com isolados de diferentes pacientes (BADDLEY e PAPPAS, 2005).

Antifúngicos azólicos associados a outros agentes antifúngicos, com diferentes mecanismos de ação, provavelmente aumentarão a eficácia no tratamento de infecções fúngicas (SHEEHAN et al., 1999), reduzindo o desenvolvimento de 
resistência a estes fármacos e, possivelmente, diminuindo a duração da terapia (NGUYEN et al., 1995). O enfoque para novos estudos de associação de fármacos, principalmente contra patógenos eucarióticos que atacam severamente pacientes imunossuprimidos deve ser voltado para regimes terapêuticos, com efeito, fungicida (DEL POETA et al., 2000).

Uma das principais razões para o uso da associação de drogas antifúngicas é a não toxicidade. Dois agentes antifúngicos podem ser usados e, portanto, em concentrações menores ao contrário da monoterapia que exige concentrações tóxicas para serem efetivas (BARCHIESI et al., 2000). Associação terapêutica realizada in vitro com anfotericina $\mathrm{B}$ e fluconazol mostrou que a combinação destas drogas permitiram o uso de baixas doses de anfotericina B sem contudo resultar em perda de uma resposta clinica (BARCHIESI et al., 2000).

Pesquisa realizada in vitro quanto à associação de drogas contra $C$. neoformans apontou para a necessidade de estudos adicionais em modelo animal para confirmar o potencial destas combinações para o tratamento da criptococcose (SERENA et al., 2005).

Atualmente a taxa de cura para infecções fungicas usando as drogas disponíveis através de monoterapia não é muito boa. Nos últimos anos, com a introdução de novas classes de drogas antifúngicas os estudos conduzem para a hipótese de que o uso de novos fármacos, em associação pode ser mais efetiva do que os mesmos usados isoladamente (POWERS, 2004). Na criptococose a associação terapêutica é mais efetiva do que a monoterapia (CISNEROS e MATÍA, 2006).

Estudo da associação da anfotericina B mais fluconazol in vitro revelou que a interação entre as drogas foi indiferente (GHANNOUM et al., 1995). POREM, 
Em outro estudo associação AMB mais FLC in vitro teve interação foi aditiva (BARCHIESI et al., 2000).

A combinação terapêutica pode ser um grande beneficio no tratamento de infecções invasivas causadas por leveduras. Todavia, o estudo da interação destas drogas in vitro ainda é muito escasso e a interpretação da concentração inibitória fracionária (FIC) pode ser contraditória devido às várias definições (TEDORSTHORST et al., 2002).

A melhora no tratamento da criptococose veio principalmente com o uso da associação de drogas antifúngicas (PARK et al., 1999; NEWTON et al., 2002). Anfotericina B, fluconazol e fluorocitosina são as drogas mais comuns no tratamento da criptococcose (SAAG et al., 2000). A combinação terapêutica em doenças causadas por C. neoformans é vantajosa (SOBEL, 2004).

Pesquisa in vivo usando camundongos BALB/c, machos, infectados experimentalmente com C. neoformans demonstrou que a associação de anfotericina B com fluconazol foi mais eficiente no tratamento destes animais do que a associação com fluorocitosina. A adição de fluorocitosina a anfotericina B e ao fluconazol não melhorou a terapia (LARSEN et al., 2004). Segundo Odds et al., (2000) o itraconazol é altamente eficaz no tratamento de infecções por C. neoformans em humanos.

Anfotericina B associada com drogas triazólicas é significantemente mais ativa do que seu uso isolado em estudo in vitro (BARCHIESI et al., 2000). Segundo os mesmos autores a associação in vivo teve efeito aditivo. Estudos da associação entre fluconazol mais anfotericina $B$ na candidemia revelou que não houve antagonismo entre as drogas (REX et al., 2003). 
O efeito da anfotericina B associada com azólicos varia de acordo com o momento da administração, sendo que a utilização de um azólico, anteriormente ao uso da anfotericina B, ocasiona antagonismo entre as duas drogas enquanto que, quando administrado simultaneamente, produz efeito sinérgico em relação ao fluconazol (VIUDES et al., 2001). O fluconazol associado a fluorocitosina mostrou maior sinergismo e maior atividade fungicida do que fluconazol associado a anfotericina B (HASSAN e ABDEL SALAM, 2005). A associação terapêutica para o tratamento da criptococose esta estabelecida baseado em evidências clinicas (JOHNSON et al., 2004).

\subsection{CORRELAÇÃO IN VITRO E IN VIVO}

A correlação dos resultados da associação AMB-FLC em criptococose experimental com os dados observados in vitro confirmou a eficácia do tratamento da doença, (BARCHIESI et al., 2000), esta eficácia foi positiva com relação aos resultados no tratamento em pacientes com infecções por leveduras. (COSTA et al., 2004).

Estudos em modelo animal quanto às novas associações de antifúngicos é crucial, esses modelos são caminhos positivos no trajeto de estudos in vitro até os pacientes (GRAYBILL, 2000). A associação entre drogas antifúngicas estão se tornando uma realidade no campo de doenças causadas por leveduras de interesse médico. As discrepâncias verificadas na correlação entre diferentes estudos in vitro e in vivo pedem que sejam adotadas metodologias padronizadas com adoção de critérios interpretativos comuns (MUKHERJEE et al., 2005). 


\section{CONCLUSÕES}

Pelos resultados obtidos no presente trabalho podemos inferir que:

6.1 de acordo com a sensibilidade in vitro apresentada prelas amostras clinicas e ambientais em relação "a anfotericina B das amostras clinicas e ambientais podemos sugerir que os isolados de ambas as fontes foram sensíveis à droga;

$6.250 \%$ das amostras clínicas de C. neoformans foram resistentes ao fluconazol, fato que sugere ser esta resistência do tipo intrínseca ou adquirida;

6.3 a resistência de $35 \%$ das amostras ambientais de C. neoformans sugere que estes isolados podem ter resistência intrínseca ao fluconazol;

6.4 nas condições estudadas constatamos que a levedura apresentou tropismo pelo cérebro;

6.5 a infecção inicial aconteceu 24 horas após a inoculação;

6.6 a meningite aguda estava instalada a partir do $7^{\circ}$ dia da inoculação; a meningite crônica a partir do $13^{\circ}$ dia, e a manifestação clinica da doença 15 após a inoculação, culminando no óbito de todos os animais;

6.7 em camundongos experimentalmente infectados, a monoterapia com anfotericina $B$ pode reduzir a sobrevida dos mesmos, enquanto que $o$ tratamento isolado com fluconazol pode prolongá-la,porem ;os animais foram ao óbito; 
6.8 o sucesso da terapia combinada depende da concentração do fluconazol na associação. Esta pode ser sinérgica para a anfotericina $B$, requerendo a metade da concentração máxima desse antifúngico;

6.9 os dados obtidos nos levam a sugerir que a melhor concentração das drogas para o tratamento da doença é AMB $1,5 \mathrm{mg} / \mathrm{kg} / \mathrm{dia}$ com FLC $30,0 \mathrm{mg} / \mathrm{kg} / \mathrm{dia}$ e que esta terapia pode ser segura, considerando-se os reduzidos efeitos secundários do poliênico quando associado;

6.10 o tratamento da criptococose deve ser iniciado logo após a suspeita de infecção,

6.11 a profilaxia com a associação AMB mais FLC pode ser benéfica; 


\section{REFERÊNCIAS BIBLIOGRÁFICAS}

$A B$ BIODISK. Etest technical guide n. 4: Antifungal susceptibility testing of yeasts. AB BIODISK, Solna, Sweden, 1993.

AKCAGLAR, S.; SEVGICAN, E.; AKALIN, H.; ENER, B.; TORE, O. Two cases of cryptococcal meningitis in immunocompromised patients not infected with HIV. Mycoses, v. 50, p. 235-238, 2007.

ALLER, A.I.; MÁRTIN-MAZUELOS, E.; GUTIÉRREZ, M.J.; BERNAL, S.; CHÁVEZ M.; RÉCIO, F.J. Comparasion of Etest and microdiluition method for antifungal susceptibility testing of cryptococcus neoformans to four antifungal agents. J. Antimicrob. Chemother., v. 46, p. 997-1000, 2000a.

ALLER, A.I.; MARTIN-MAZUELOS, E.; LOZANO, F.; GOMEZ-MATEOS, J.; STEELE-MOORE, L.; HOLLOWAY, W.J.; GUTIÉRREZ, M.J.; RECIO, F.J.; ESPINEL-INGROFF, A. Correlation of Fluconazole MICs with Clinical Outcome in Cryptococcal Infection. Antimicrob. Agents. Chemother., v. 44, p. 1544-1548, 2000b.

ALLER, A.I.; CLARO, R.; CASTRO, C.; SERRANO, C.; COLOM, M.F.; MARTÍNMAZUELOS, E. Antifungal Susceptibility of Cryptococcus neoformans Isolates in HIV-Infected Patients to Fluconazole, Itraconazole and Voriconazole in Spain: 19941996 and 1997-2005. Chemotherapy, v. 35, p. 300-305, 2007.

ALVES, S.H; OLIVEIRA, L.T; COSTA, J.M; LUBECK, I; CASALI, A.K; VAINSTEIN, M.H. In vitro susceptibility antifungal agents of clinical and environmental Cryptococcus neoformans isolated in southern of Brazil. Rev. Inst. Med. Trop. S. Paulo, v. 43, p. 5, 2001.

AOKI, Y.; YOSHIHARA, F.; KONDOH, M.; NAKAMURA, Y.; NAKAYAMA, N.; ARISAWA, M. Ro 09-1470 Is a Selective Inhibitor of P-450 Lanosterol C-14 Demethylase of Fungi. Antimicrob. Agents. Chemother., v. 37, p. 2662-2667, 1993.

De acordo com:

ASSOCIAÇÃO BRASILEIRA DE NORMAS TÉCNICAS. NBR 6023:

Informação e documentação: referências: elaboração. Rio de Janeiro, 2002.

NATIONAL LIBRARY OF MEDICINE. List of journals indexed in Index Medicus. 2006. Available from:

http://www.ncbi.nlm.nih.gov/entrez/; http://www.nlm.nih.gov/tsd/serials/lij.html 
ARAÚJO, I.B.; BRITO, C.R.; URBANO, I.A.; DOMINICI, V.A.; SILVA FILHO, M.A.; SILVEIRA, W.L.; DAMASCENO, B.P.; MEDEIROS, A.C.; EGITO, E.S. Similarity between the in vitro activity and toxicity of two different fungizone ${ }^{\mathrm{TM}} /$ lipofundin $^{\mathrm{T}}$ admixtures. Acta. Cir. Brás., v. 20, p. 129-133, 2005.

BADDLEY, J.W.; PAPPAS, P.G. Antifungal combination therapy: clinical potential. Drugs. v. 65, p. 1461-1480, 2005.

BARCHIESI, F.; R. HOLLIS, S. A. MESSER, G. SCALISE, M. G. RINALDI.; M. A. PFALLER. Electrophoretic karyotype and in vitro antifungal susceptibility of Cryptococcus neoformans isolates from AIDS patients. Diagn. Microbiol. Infect. Dis. v. 23, p. 99-103, 1995.

BARCHIESI, F.; SCHIMIZZI, A.M.; CASELLI, F.; NOVELLI, A.; FALLANI, S.; GIANNINI. D.; ARZENI, D.; DI CESARE, S.; DI FRANCESCO, L.F.; Fortuna, M.; GIACOMETTI, A.; CARLE, F.; MAZZEI, T.; SCALISE, G. Interactions between Triazoles and Amphotericin B against Cryptococcus neoformans. Antimicrob. Agents. Chemother., v. 44, p. 2435-2441, 2000.

BARÓ, T.; TORRES-RODRÍGUEZ, J.M.; MORERA, Y. ALÍA, C.; LÓPEZ, O.; MÉNDEZ, R. Serotyping of Cryptococcus neoformans Isolates from Clinical and Environmental Sources in Spain. J. Clin. Microbiol., v. 37, p.1170-1172, 1999.

BARONI, F. A. Ocorrência de Cryptococcus neoformans em excretas de pombos localizadas em torres de igrejas na cidade do Rio de Janeiro: Fatores de virulência e sensibilidade aos antifúngicos. 2001. 232f. Tese (Doutorado em Ciências), Instituto de Ciências Biomédicas da Universidade de São Paulo, São Paulo, 2001.

BARONI, F.A.; GONÇALVES DA SILVA, E; VIANI, F.C.; OLIVEIRA, M.T.B.; RIVERA, I.N.G.; GAMBALE, W.; PAULA, C.R Cryptococcus neoformans strains isolated from church towers in Rio de Janeiro city, RJ, Brazil. Rev. Inst. Med. Trop. S. Paulo. v. 48, p. 71-75, 2006.

BODEY, G. P. Infection in cancer patients: a continuing association Am. J. Med., v. 81, p. 11-26, 1986.

BRANDT, M.E.; PFALLER. M.A.; HAJJEH, R.A.; HAMILL, R.J.; PAPPAS, P.G.; REINGOLD, A.L.; RIMLAND, D.; WARNOCK, D.W. FOR THE CRYPTOCOCCAL DISEASE ACTIVE SURVEILLANCE GROUP. Trends in Antifungal Drug Susceptibility of Cryptococcus neoformans Isolates in the United States: 1992 to 1994 and 1996 to 1998. Antimicrob. Agents. Chemother., v. 45, p. 3065-3069, 2001.

BROUWER, A.; RAJANUWONG, A.; CHIERAKUL, W.; GRIFFIN G.; LARSEN, R.; WHITE, N.;HARRISON, T. Combination antifungal therapies for HIV-associated cryptococcal meningitis: a randomised trial. Lancet, v. 363, p. 1764-1767, 2004. 
BUCHANAN, K. L.; MURPHY, J.W. What makes Cryptococcus neoformans a pathogen? Emerg. Infect. Dis., v. 4, p. 71- 83, 1998.

CAFARCHIA, C.; ROMITO, D.; IATTA, R.; CAMARDA, A.; MONTAGNA, M.T.; OTRANTO, D. Role of birds of prey as carriers and spreaders of Cryptococcus neoformans and other zoonotic yeasts. Med. Mycol., v. 44, p. 485-492, 2006.

CALVO, B.M.; COLOMBO, A. L.; FISCHMAN, O.; SANTIAGO, A.; THOMPSON, L.; LAZERA, M.S.; TELLES, F.; FUKUSHIMA, K.; NISHIMURA, K.; TANAKA, R.; MYIAJY, M.; MORETTI-BRANCHINI, M.L. Antifungal Susceptibilities, Varieties, and Electrophoretic Karyotypes of Clinical Isolates of Cryptococcus neoformans from Brazil, Chile, and Venezuela. J. Clin. Microbiol., v. 39, p. 2348-2350, 2001.

CANTEY, P.T.; STEPHENS, D.S.; RIMLAND, D. Prevention of cryptococcosis in HIV-infected patients with limited access to highly active antiretroviral therapy: evidence for primary azole prophylaxis. Hiv. Med., v. 6, p. 253-259, 2005.

CAPILLA, J.; MAFFEI, C.M.L.; CLEMONS, K.V.; SOBEL, R.A.; STEVENS,D.A. Experimental systemic infection with Cryptococcus neoformans var. grubii and Cryptococcus gattii in normal and immunodeficient mice. Med. Mycol., v. 44, p. 601610, 2006.

CASADEVALL, A.; PERFECT, J.R. Cryptococcus neoformans. Washington: American Society for Microbiology, 1998.

CASALI, A. K.; GOULART,L.; KMETZSCH, L.; ROSA, S.; RIBEIRO, ANGELA, A.M.; AMARAL, A. Al.; ALVES, S.H.; SCHRANK, A.; MEYER W.; VAINSTEIN, M.H. Molecular typing of clinical and environmental Cryptococcus neoformans isolates in the Brazilian state Rio Grande do Sul. FEMS Yeast Res., v. 3, p. 405-415, 2003.

CHANG, H.C.; CHANG, J.J.; CHAN, S.H.; HUANG, A.H.; WU, T.L.; LIN,M. C.; CHAN, T.C.G. Evaluation of Etest for Direct Antifungal Susceptibility Testing of Yeasts in Positive Blood Cultures. J. Clin. Microbiol., v. 39, p. 1328-1333, 2001.

CHANG, Y.C.; STINS, M.F.; MCCAFFERY, M.J.; MILLER, G.F.; PARE, D.R.; DAM, T.; PAUL-SATYASEE, M.; KIM, K.S.; KWON-CHUNG, K.J. Cryptococcal Yeast Cells Invade the Central Nervous System via Transcellular Penetration of the Blood-Brain Barrier . Infect. Immun., v. 72, p. 4885-4995, 2004a.

CHANG, W.N.; HUANG, C.R.; LEI, C.B.; LEE, P.Y. CHIEN, C.C.; CHANG, H.W.; CHANG, C.S.; LU, C.H. Serotypes of clinical cerebrospinal fluid Cryptococcus neoformans isolates from southern Taiwan and their in vitro susceptibilities to amphotericin B, fluconazole, and voriconazole. Jpn. J. Infect. Dis., v. 57, p. 113-115, 2004b.

CHANG, L.W.; PHIPPS, W.T.; KENNEDY, G.E.; RUTHERFORD, G.W. Antifungal interventions for the primary prevention of cryptococcal disease in adults with HIV. Cochrane Database Syst. Rev., v. 20, cd 004776, 2005. 
CHARLIER, C.; CHRETIEN, F.; BAUDRIMONT, M.; MORDELET, E.; LORTHOLARY, O.; DROMER, F. Capsule Structure Changes Associated with Cryptococcus neoformans Crossing of the Blood- Brain Barrier. Am. J. Pathol., v. 166, p. 421-432, 2005.

CHEE, H.Y.; LEE, K.B. Isolation of Cryptococcus neoformans var. grubii (serotype A) from pigeon droppings in Seoul, Korea. J. Microbiol., v. 43, p. 469-472, 2005.

CHEN, S.C.A. Cryptocococosis in Australasia and the treatment of cryptococcal and other fungal infections with lipossomal amphotericin B. J. Antimicrob. Chemother, v. 49 Suppl S1, p. 57-61, 2002.

CHRÉTIEN, F.; LORTHOLARY, O.; KANSAU, I.; NEUVILLE, S.; GRAY, F.; DROMER, F. Pathogenesis of Cerebral Cryptococcus neoformans infection after fungemia. J. Infect. Dis., v. 186, p. 522-530, 2002.

CISNEROS HERREROS, J.M.; CORDERO MATÍA, E. Therapeutic armamentarium against systemic fungal infections. Clin. Microbiol. Infect., v. 12 suppl 7, p. 53-64, 2006.

COSTA, M.; PASSOS, S.S.; MIRANDA, A.T.B.; ARAÚJO, R.S.C.; PAULA, C. R.; SILVA, M.R.R. Correlation of in vitro itraconazole and fluconazole susceptibility with clinical outcome for patients with vulvovaginal candidiasis. Mycopathologia, v. 157, p. 43-47, 2004.

CRISSEY, J.T.; LANG, H.; PARISH, L.C. Manual of Medical Mycology, Spain, Blackwell Science, 1995.

CRUZ, M.V.N. Fenótipos e Sensibilidade aos Antifúngicos de amostras Clínicas e Ambientais de Cryptococcus neoformans. 2004. 107f. Dissertação (Mestrado em Ciências), Instituto de Ciências Biomédicas da Universidade de São Paulo, São Paulo, 2004.

DANNAOUI, E.; ABDUL, M.; ARPIN, M.; MICHEL-NGUYEN, A.; PIENS, M.A.; FAVEL, A.; LORTHOLARY, O.; DROMER, F.; THE FRENCH CRYPTOCOCCOSIS STUDY GROUP. Antimicrob. Agents. Chemother., v. 50, p. 2464-2470, 2006.

DELGADO, A.C.N.; TAGUCHI, H.; MIKAMI, Y.; MYIAJY, M.; VILLARES, M.C.B.; MORETTI, M.L. Human cryptococcosis: relationship of environmental and clinical strains of Cryptococcus neoformans var. neoformans from urban and rural areas. Mycopathologia, v. 159, p. 7-11, 2005.

DEL POETA, M.; CRISTINA CRUZ, M.; CARDENAS, M.E.; PERFECT, J.R.; HEITMAN, J. Synergistic Antifungal Activities of Bafilomycin A1, Fluconazole, and the Pneumocandin MK-0991/Caspofungin Acetate (L-743,873) with Calcineurin Inhibitors FK506 and L-685,818 against Cryptococcus neoformans. Antimicrob. Agents. Chemother., v. 44, p. 739-746, 2000. 
DENNING, D.W.; ARMSTRONG, R.W.; LEWIS, B.H.; STEVENS, D.A. Elevated cerebrospinal fluid pressures in patients with cryptococcal meningitis and acquired immunodeficiency syndrome. Am. J. Med., v. 91, p. 267-272, 1991.

DERAY G. Amphotericin B nephrotoxicity. J. Antimicrob. Chemother., v. 49 Suppl S1, p. 37-41, 2002.

DIAS, A.L.; MATSUMOTO, F.E.; MELHEM, M.S.; DA SILVA, E.G.; AULER, M.E.; DE SIQUEIRA, A.M.; PAULA, C.R. Comparative analysis of Etest and broth microdilution method (AFST-EUCAST) for trends in antifungal drug susceptibility testing of Brazilian Cryptococcus neoformans isolates. J. Med. Microbiol., v. 55, p. 1693-1699, 2006.

DING, J.S.C.; BAUER, M.; DIAMOND, D.M.; LEAL, M.A.E.; JOHNSON, D.; WILLIAMS, B.; THOMAS, A.M.; NAJVAR, L.; GRAYBILL, J.R.; LARSEN, R. Effect of Severity of Meningitis on Fungicidal Activity of Flucytosine Combined with Fluconazole in a Murine Model of Cryptococcal Meningitis. Antimicrob. Agents. Chemother., v. 14. p. 1589-1593, 1997.

DROMER, F.; PERRONNE, C.; BARGE, J.; VILDE, J.L.; YENI, P. Role of IgG and complement component $\mathrm{C} 5$ in the initial course of experimental cryptococcosis. Clin. Exp. Immunol., v. 78, p. 412-417, 1989.

ESPINEL-INGROFF, A. Clinical relevance of antifungal resistance. Infect. Dis. Clin. N. Amer., v. 11, p.929-944, 1997.

FERNANDES, O.F.L.; PASSOS, X.S.; SOUZA, L.K.H.; MIRANDA, A.T.B.; CERQUEIRA, C.H.P.V.; SILVA, M.R.R. In vitro susceptibility characteristics of Cryptococcus neoformans varieties from AIDS patients in Goiânia, Brazil. Mem. Inst. Oswaldo Cruz, v. 98, p. 839-841, 2003.

FORD, B. J. AIDS and Africa. Biologist, v. 47, p.224, 2000.

FILIÚ, W.F.OL.; WANKE, B.; AGÜENA, S.M.; VILELA, V.O.; MACEDO, R. C.L; LAZÉRA, M. Avian habitats as sources of Cryptococcus neoformans in the city of Campo Grande, Mato Grosso do Sul, Brazil. Rev. Soc. Brás. Méd. Trop., v. 35, p. 591-595, 2002.

FRANZOT, S.P.; SALKIN, I.F.; CASADEVALL, A. Cryptococcus neoformans var. grubii: separate varietal status for Cryptococcus neoformans serotype A isolates. J. Clin. Microbiol., v. 37, p. 838-840, 1999.

GARCIA-HERMOSO, D.; JANBON, G.; DROMER, F. Epidemiological evidence for dormant Cryptococcus neoformans infection. J. Clin. Microbiol., v. 37, p. 32043209, 1999.

GARCÍA-MARTOS, P.; NOVAL, J.F.; GARCÍA-TAPIA, A.; MARÍN, P.; PUERTO, J.L.; SEPÚLVEDA, A. Sensibilidad a antifúngicos de especies de Cryptococcus de interés clínico. Méd. Clin. (Barc)., v. 119, p. 211-213, 2002. 
GEORGOPAPADAKOU, N.H.; WALSH, T.J. Human mycoses: drugs and targets for emerging pathogens. Science, v. 264, p. 371-373, 1994.

GEORGOPAPADAKOU, N.H.; WALSH, T.J. Antifungal Agents: Chemotherapeutic Targets and Immunologic Strategies. Antimicrob. Agents. Chemother., v. 40, p. 279-291, 1996.

GHANNOUM, M.A.; FU, Y.; IBRAHIM, A.S.; MORTARA, L.A.; SHAFIQ, M.C.; EDWARDS, J.E.; CRIDDLE, R.S. In Vitro Determination of Optimal Antifungal Combinations against Cryptococcus neoformans and Candida albicans. Antimicrob. Agents. Chemother., v. 39, p. 2459-2465, 1995.

GRAYBILL, J.R. The role of murine models in the development of antifungal therapy for systemic mycoses. Drug. Resist. Updat., v. 3, p. 364-383, 2000.

HASSAN, A.; ABDEL-SALAM. In vitro susceptibility of Cryptococcus neoformans clinical isolates from Egypt to seven antifungal drugs. Mycoses, v. 48, p. 327-332, 2005.

HOSSAIN, M.A.; MUKHERJEE, P.K.; REYES G.; LONG, L.; GHANNOUM, M.A. Effects of fluconazole singly and in combination with 5-fluorocytosine or amphotericin $B$ in the treatment of cryptococcal meningoencephalitis in an intracranial murine model. J. Chemother., v. 14, p. 351-360, 2002.

HSUEH, P.R.; LAU, Y.J.; CHUANG, Y.C.; WAN, J.H.; HUANG, W.K.; SHYR, J.M.; YAN, J.J.; YU, K.W.; WU, J.J.; KO, W.C.; YANG, Y.C.; LIU, Y.C.; TENG, L.J.; LIU, C.Y.; LUH, K.T. Antifungal Susceptibilities of Clinical Isolates of Candida Species, Cryptococcus neoformans, and Aspergillus Species from Taiwan: Surveillance of Multicenter Antimicrobial Resistance in Taiwan Program Data from 2003. Antimicrob. Agents. Chemother., v. 49, p. 512-517, 2005.

IKEDA, R.; SHINODA, T.; FUKAZAWA, Y.; KAUFMAN, L. Antigenic Characterization of Cryptococcus neoformans Serotypes and Its Application to Serotyping of Clinical Isolates. J. Clin. Microbiol., v. 16, p. 22-29, 1982.

JUST-NUBLING, G. Therapy of candidiasis and cryptococcosis in AIDS. Mycoses, v.37 suppl 2, p. 56-63, 1994.

JOHNSON, M.D.; MACDOUGALL, C.; OSTROSKY-ZEICHNER, L.; PERFECT, J.R.; REX, J.H. Combination Antifungal Therapy. Antimicrob. Agents. Chemother., v. 48, p. 693-715, 2004.

KIM, K.S. Pathogenesis of bacterial meningitis: from bacteria to neuronal injury. Nat. Rev. Neurosci., v. 4, p.376-385, 2003.

KLEPSER, M.E.; WOLFE, E.J.; PFALLER, M.A. Antifungal pharmacodynamic characteristics of fluconazole and amphotericin B against Cryptococcus neoformans. J. Antimicrob. Chemoter., v. 41, p. 397-401, 1998. 
KOBAYASHI, C.C. B.A.; SOUZA, L. K. H.; FERNANDES, O. F.L.; BRITO, S. C. A.; SILVA, A.C.; SOUSA, E.D.; SILVA, M.R.R. Characterization of Cryptococcus neoformans isolated from urban environmental sources in Goiânia, Goiás State, Brazil. Rev. Inst. Med. trop. S. Paulo, v. 47, p. 203-207, 2005.

KOUAME-ASSOUAN, A.E.; COWPPLI-BONY, P.; AKA-ANGHUI, D.E.; ASSI, B.; DOUMBIA, M.; DIALLO, L.; ADJIEN, K.C.; AKANI, E.; SONAN, T.; DIAGANA, M. Two cases of cryptococcal meningitis revealed by an ischemic stroke. Bull. Soc. Pathol. Exot., v. 100, p. 15-16, 2007.

KOVACS, J.A.; KOVACS, A.A.; POLIS, M. Cryptococcosis in the acquire immunodeficiency syndrome. Ann. Inter. Med., v.103, p. 533-538, 1985.

KURTZMAN, C.P.; FELL, J.W. The yeasts: a taxonomic study. $4^{\mathrm{a}}$ ed, Amsterdam: Elsevier Science, 1998. 1055p.

LACAZ, C.S.; NEGRO, G. Drogas antifúngicas. Terapêutica das micoses. In: LACAZ, C.S.; PORTO, E.; MARTINS, J.E.C. Micologia médica fungos, actinomicetos e algas de interesse médico. São Paulo : Savier, 1991.

LACAZ, C.S.; PORTO, E.; MARTINS, J.E.C.; VACCARI-HEINS, E.M.; DE MELO, N.T. Tratado de Micologia Médica Lacaz. São Paulo: Sarvier, 2002.

LAKSHMI, V.; SUDHA, T.; TEJA, V.D.; UMABALA, P. Prevalence of central nervous system cryptococcosis in human immunodeficiency virus reactive hospitalized patients. Indian. J. Med. Microbiol., v. 25, p. 146-149, 2007.

LARSEN, R.A.; BAUER, M.; THOMAS, A. M.; GRAYBILL, J. Amphotericin B and Fluconazole, a Potent Combination Therapy for Cryptococcal Meningitis. Antimicrob. Agents. Chemother., v. 48, p. 985-991, 2004.

LARSEN, R.A., BAUER, M., THOMAS, A.M., SANCHEZ, A., CITRON, D., RATHBUN, M.; HARRISON, T.S. Correspondence of in vitro and in vivo fluconazole dose-response curves for Cryptococcus neoformans. Antimicrob. Agents. Chemother., v. 49, p. 3297- 3301, 2005.

LEITE, A.G.; VIDAL, J.E.; BONASSER, FILHO F.; NOGUEIRA, R.S.; OLIVEIRA, A.C. Cerebral Infarction Related to Cryptococcal Meningitis in an HIV-Infected Patient: Case Report and Literature Review. Braz. J. Infect. Dis., v. 8, p. 175-179, 2004.

LIZARAZO, J.; LINARES, M.; BEDOUT, C.; RESTREPO, Á.; AGUDELO, C.I.; CASTAÑEDA, E.; GRUPO COLOMBIANO PARA EL ESTUDIO DE LA CRIPTOCOCOSIS. Estudio clínico y epidemiológico de la criptococosis en Colombia: resultados de nueve años de la encuesta nacional, 1997-2005. Biomédica, v. 27, p. 94-106, 2007. 
LITVINTSEVA, A.P.; KESTENBAUM, L.; VILGALYS, R.; MITCHELL, T.G. Comparative Analysis of Environmental and Clinical Populations of Cryptococcus neoformans. J. Clin. Microbiol., v. 43, p. 556-564, 2005.

LÓPEZ-MEDRANO, F.; DÍAZ-PEDROCHE, C.; LUMBRERAS, C.; AGUADO, J.M. Utilidad de la amfotericina $B$ liposomal para la prevención de la infección fúngica en receptores de un trasplante de órgano sólido. Rev. Esp. Quimioterap., v. 18, p. 1420, 2005.

LORTHOLARY, O.; NICOLAS, M.; SOREDA, S.; IMPROVISI, L.; RONIN, O.; PETITJEAN, O.; DUPONT, B.; TOD, M.; DROMER, F. Fluconazole, with or without dexamethasone for experimental cryptococcosis: impact of treatment timing. $\mathbf{J}$. Antimicrob. Chemoter., v. 43, p. 817-824, 1999.

LOZANO-CHIU, M.; PAETZNICK, V.L.; GHANNOUM, M.A.; REX J.H. Detection of Resistance to Amphotericin B among Cryptococcus neoformans Clinical Isolates: Performances of Three Different Media Assessed by Using E-Test and National Committee for Clinical Laboratory Standards M27-A Methodologies. J. Clin. Microbiol., v. 36, p. 2817-2822, 1998.

LUMBRERAS, C.; LIZASOAIN, M.; AGUADO, J.M. Antifúngicos de uso sistémico. Enferm. Infec. Microbiol. Clin., v. 21, p. 366-379, 2003.

MAFFEI, C.M.L.; MIRELS, L.F.; SOBEL, R.A.; CLEMONS, K.V.; DAVID, A. S. Cytokine and Inducible Nitric Oxide Synthase mRNA Expresión during Experimental Murine Cryptococcal Meningoencephalitis. Infect. Immun., v. 72, p. 2338-2349, 2004.

MARTINEZ, L. R.; GARCIA-RIVERA, J.; CASADEVALL, A. Cryptococcus neoformans var. neoformans (Serotype D) Strains Are More Susceptible to Heat than C. neoformans var. grubii (Serotype A) Strains. J. Clin. Microbiol., v. 39, p. 33653367, 2001.

MATAR, M.J.; OSTROSKY-ZEICHNER, L.; PAETZNICK, V.L.; RODRIGUEZ, J. R.; CHEN, E.; REX, J.H. Correlation between E-Test, Disk Diffusion, and Microdilution Methods for Antifungal Susceptibility Testing of Fluconazole and Voriconazole. Antimicrob. Agents. Chemother., v. 47, p. 1647-1651, 2003.

MAXWELL, M.J.; MESSER, S.A.; HOLLIS, R.J.; DIEKEMA, D.J.; PFALLER M.A. Evaluation of Etest Method for Determining Voriconazole and Amphotericin B MICs for 162 Clinical Isolates of Cryptococcus neoformans. J. Clin. Microbiol., v. 41, p. 97-99, 2003.

MITCHELL TG, PERFECT J.R. Cryptococcosis in the era of AIDS-100 years after the discovery of Cryptococcus neoformans. Clin. Microbiol. Rev., v. 8, p. 515-548, 1995.

MONTENEGRO, H.; PAULA, C.R. Environmental isolation of Cryptococcus neoformans var. gattii and C. neoformans var. neoformans in the city of São Paulo, Brazil, Med. Mycol., v. 38, p. 385-390, 2000. 
MOREIRA, T.A.; FERREIRA, M.S.; RIBAS, R.M.; BORGES, A.S. Criptococose: estudo clínico-epidemiológico, laboratorial e das variedades do fungo em 96 pacientes. Rev. Soc. Bras. Inst. Med. Trop., v. 39, p. 255-258, 2006.

MUKHERJEE, P. K.; SHEEHAN, 1 D.J.; HITCHCOCK, C.A.; GHANNOUM, M.A. Combination Treatment of Invasive Fungal Infections. Clin. Microbiol. Rev., v. 18, p. 163-194, 2005.

NATIONAL COMMITTEE FOR CLINICAL LABORATORY STANDARDS. 2002. Reference method for broth dilution antifungal susceptibility testing of yeasts; approved standard, 2nd ed. M27-A2. National Committee for Clinical Laboratory Standards, Wayne, Pa.

NEWTON, P. N.; H., THAI LE; N. Q., TIP; J. M., SHORT; W., CHIERAKUL; A., RAJANUWONG; P., PITISUTTITHUM; S., CHASOMBAT; B., PHONRAT; W., MAEKA-NANTAWAT; R., TEAUNADI; D. G., LALLOO; N. J., WHITE. A randomized, double blind, placebo-controlled trial of acetazolamide for the treatment of elevated intracranial pressure in cryptococcal meningitis. Clin. Infect. Dis., v. 35, p. 769-772, 2002.

NGUYEN, M.H.; BARCHIESI, F.; YU, V.L.; RINALDI, M.G. In vitro evaluation of combination of fluconazole and flucytosine against Cryptococcus neoformans var. neoformans. Antimicrob. Agents. Chemother., v. 39, p.1691-1695, 1995.

NGUYEN, M.H.; YU, C.Y. In vitro comparative efficacy of voriconazole and itraconazole against fluconazole-susceptible and resistant Cryptococcus neoformans isolates. Antimicrob. Agents. Chemother., v. 42, p. 471-472, 1098.

NUNEZ, M.; PEACKOC, J.E. Jr.; CHIN, R, Jr. Pulmonary cryptococcosis in the immunocompetent host. Therapy with oral fluconazole: a report of four cases and a review of the literature. Chest, v. 118, p. 527-534, 2000.

ODDS, F.C.; ORIS, M.; VAN DORSSELAER, P.; GERVEN, F.Activities of an Intravenous Formulation of Itraconazole in Experimental Disseminated Aspergillus, Candida, and Cryptococcus Infections. Antimicrob. Agents. Chemother., v.44, p. 3180-3183, 2000.

ORNI-WASSERLAUF, R.; IZKHAKOV, E.; SIEGMAN-IGRA,Y. BASH, E.; POLACHECK, I.; GILAD, M. Fluconazole resistant Cryptococcus neoformans isolated from an immunocompetent patient without prior exposure to fluconazole. Clin. infect. Dis., v. 29, p. 1592-1593, 1999.

PAL, M. Pathogenicity of environmental strains of Cryptococcus neoformans var neoformans in murine model. Rev. Iberoam. Micol., 22, 2005.

PAPPALARDO, M.C.S.M.; MELHEM, M.S.C. CRYPTOCOCCOSIS: A REVIEW OF THE BRAZILIAN EXPERIENCE FOR THE DISEASE. Rev. Inst. Med. Trop. S. Paulo., v. 45, p. 299-305, 2003. 
PARK, M. K.; D. R., HOSPENTHAL; J. E., BENNETT. Treatment of hydrocephalus secondary to cryptococcal meningitis by use of shunting. Clin.Infect. Dis., v. 28, p. 629-633, 1999.

PEDROSO, R.S.; FERREIRA, J.C.; CANDIDO, R.C. In vitro susceptibility to antifungal agents of environmental Cryptococcus spp. isolated in the city of Ribeirão Preto, São Paulo, Brazil. Mem. Inst. Oswaldo Cruz., v. 105, p. 239-243, 2006.

PERFECT, J. R.; D. T. DURAK. Treatment of experimental cryptococcal meningitis with amphotericin B, 5-fluorocytosine and ketoconazole. J. Infect. Dis. v. 146, p.429435, 1982.

PERKINS, A.; GOMEZ-LOPEZ, A.; MELLADO, E.; RODRIGUEZ-TUDELA, J.L; CUENCA-ESTRELLA, M. Rates of antifungal resistance among Spanish clinical isolates of Cryptococcus neoformans var. neoformans. J. Antimicrob. Chemoter., v. 56, p. 1144-1147, 2005.

PETROU, M.A; SHANSON, D.D; Susceptibility of Cryptococcus neoformans by The NCCLS and Etest methods using five defined media. J. Antimicrob. Chemoter., v. 46, p. 815-818, 2000.

PFALLER, M.A.; ZHANG, J.; MESSER, S.A.; BRANDT, M.E.; HAJJEH, R.A.; JESSUP, C.J.; TUMBERLAND, M.; MBIDDE, E.K.; GHANNOUM, M.A. In vitro activities of voriconazole, fluconazole, and itraconazole against 566 clinical isolates of Cryptococcus neoformans from the United States and Africa. Antimicrob. Agents. Chemother., v. 43, p.169-71, 1999.

PFALLER, M.A.; MESSER, S.A.; BOYKEN, L.; RICE, C.; TENDOLKAR S.; HOLLIS, R.J.; DIEKEMA, D. J. Evaluation of the NCCLS M44-P Disk Diffusion Method for Determining Susceptibilities of 276 Clinical Isolates of Cryptococcus neoformans to Fluconazole. J. Clin. Microbiol., v. 42, p. 380-383, 2004.

PFALLER, M.A.; MESSER, S.A; BOYKEN, L.; RICE, C.; TENDOLKAR, S.; HOLLIS, R.J.; DOERN, G.V.; DIEKEMA, D.J. Global Trends in the Antifungal Susceptibility of Cryptococcus neoformans (1990 to 2004). J. Clin. Microbiol., v. 43, p. 2163-2167, 2005.

PFISTER, H.W.; BORASIO, G.D.; DIRNAGL, U.; BAUER, M. \& EINHÄUPL, K.M. Cerebrovascular complications of bacterial meningitis in adults. Neurology., v. 42, p. 1497-1504, 1992.

PITISUTTITHUM, P.; S., TANSUPHASAWADIKUL; A. J., SIMPSON; P. A., HOWE and N. J., WHITE. A prospective study of AIDS-associated cryptococcal meningitis in Thailand treated with high-dose amphotericin B. J. Infect, v. 43, p. 226-233, 2001.

POWERS, J.H.; Considerations in clinical trials of combination antifungal therapy. Clin. Infect. Dis, Suppl 4, p. S228-235, 2004.

POWDERLY, W. G.; SAAG M. S.; CLOUD G. A.; ROBINSON P.; MEYER R. D.; JACOBSON J. M.; J. R. GRAYBILL; A. M. SUGAR; V. J. MCAULIFFE; S. E. 
FOLLANSBEE et al. A controlled trial of fluconazole or amphotericin B to prevent relapse of cryptococcal meningitis in patients with the acquired immunode- ficiency syndrome. N. Engl. J. Med. v. 326, p. 793-798, 1992.

POWDERLY, W.G. Cryptococcal meningitis in HIV-infected patients. Curr. infect. Dis. Rep. v. 2, p. 352-357, 2000.

QUINTERO, E.; CASTAÑEDA, E.; RUIZ, A. Distribución ambiental de Cryptococcus neoformans en el departamento de Cundinamarca-Colombia. Rev. Iberoam. Micol., v. 22, p. 93-98, 2005.

REIMÂO, J.Q.; DRUMMOND, E.D.; TERCETI, M.S.; LYON, J.P.; FRANCO, M.C.; SIQUEIRA, A.M. Isolation of Cryptococcus neoformans from hollows of living trees in the city of Alfenas, MG, Brazil. Mycoses, v. 50, p. 261-264, 2007.

REX, J.H.; PAPPAS, P.G.; KARCHMER, A.W.; SOBEL, J.; EDWARDS, J.E.; HADLEY, S.; BRASS, C.; VAZQUEZ, J.A.; CHAPMAN, S.W.; HOROWITZ, H.W.; ZERVOS, M.; MCKINSEY, D.; LEE, J.; BABINCHAK, T.; ROBERT W.; BRADSHER, R.W.; CLEARY, J.D.; COHEN, D.M.; DANZIGER, L.; GOLDMAN, M.; GOODMAN, J.; HILTON, E.; HYSLOP, N.E.; KETT, D.H.; LUTZ, J.; RUBIN, R. H.; SCHELD, W.M.; SCHUSTER, M.; SIMMONS, B.; STEIN, D.K.; WASHBURN, R.G.; MAUTNER, L.; CHU, TENG-CHIAO; PANZER, H.; ROSENSTEIN, R.B. AND BOOTH, J.A, FOR THE NATIONAL INSTITUTE OF ALLERGY AND INFECTIOUS DISEASES MYCOSES STUDY GROUPA. A Randomized and Blinded Multicenter Trial of High-Dose Fluconazole plus Placebo versus Fluconazole plus Amphotericin B as Therapy for Candidemia and Its Consequences in Nonneutropenic Subjects. Clin. Infect. Dis., v.36, p. 1221-1228, 2003.

RIBEIRO, M.; PAULA, C.R.; PERFECT J.R.; COX, G.M. Phenotypic and genotypic evaluation of fluconazole resistance in vaginal Candida strains isolated from HIVinfected women from Brazil. Med. Micol., v. 43, p. 647-650, 2005.

RICHARDSON, M.D.; WARNOCK, D.W. Fungal infection - Diagnosis and management. London : Blackwell, 1993. Cap.3: Antifungal drugs: 17-43.

RIVAS, P. \& SERRANO, R.Q. Utilidad Clínica de las Pruebas de Susceptibilidad Antimicótica. REVISTA COLOMBIANA DE CANCEROLOGÍA, p. 34-42, 2003.

RIVERA, J.; FELDMESSER, M.; CAMMER, M.; CASADEVALL, A. Organdependent variation of capsule thickness in Cryptococcus neoformans during experimental murine infection. Infect. Immun., v. 66, p. 5027-5030, 1998.

ROBINSON, P. A.; M. BAUER; M. A., LEAL; S. G., EVANS; P. D., HOLTOM; D. A., DIAMOND; J. M., LEEDOM; R. A., LARSEN. Early mycological treatment failure in AIDS-associated cryptococcal meningitis. Clin. Infect. Dis., v. 28, p. 82-92, 1999.

RODRIGUES-TUDELA, J.L; MARTIN-DIEZ, F; CUENCA-ESTRELLA, M; RODERO, L; CARPINTEIRO, Y; GORGOJO, B. Influence of shaking on antifungal susceptibility testing of Cryptococcus neoformans: comparison of the NCCLS standard M27A 
medium, buffered yeast nitrogen base, and RPMI-2\% glucose. Antimicrob. Agents. Chemother., v.44, p. 400-404, 2000.

RODRIGUES-TUDELA, J.L. Method for determination of minimum inhibitory concentration (MIC) by broth dilution of Fermentative yeast. In: SUBCOMMITTEE ON ANTIFUNGAL SUSCEPTIBILITY TESTING (AFST) of the ESCMID European COMMITTEE FOR ANTIMICROBIAL SUSCEPTIBILITY TESTING, Munich, Germany: EUCAST, Jun 2002.

ROGERS, T.R. Antifungal drug resistance: limited data, dramatic impact? Int. J. Antimicrob. Agents., v. 27. p. 7-11, 2006.

RUBIN, L.L. The cell biology of the blood-brain barrier. Annu. Rev. Neurosci., v. 22, p. 11-28, 1999.

SAAG, M. S.; J. R. GRAYBILL; R. A. LARSEN; P. G. PAPPAS; J. R. PERFECT; W. G. POWDERLY; J. D. SOBEL.; W. E. DISMUKES. Practice guidelines for the management of cryptococcal disease. Clin. Infect. Dis, v. 30, p. 710-718, 2000.

SANGLARD, D. Clinical relevance of mechanisms of antifungal drug resistance in yeasts. Enferm. Infecc. Microbiol. Clin., v. 20, p. 462-70, 2002.

SANATI, H.; MESSER S. A.; PFALLER M.; WITT M., ESPINEL-INGROFF A.; GHANNOUM. M. Multicenter evaluation of broth microdilution method for susceptibility testing of Cryptococcus neoformans against fluconazole. J. Clin. Microbiol., v. 34, p.1280-1282, 1996.

SERENA, C.; FERNÁNDEZ-TORRES, B.; PASTOR, F.J.; TRILLES, L.; LAZÉRA, M.S.; NOLARD, N.; GUARRO, J. In Vitro Interactions of Micafungin with Other Antifungal Drugs against Clinical Isolates of Four Species of Cryptococcus. Antimicrob. Agents Chemother., v. 49, p. 2994-2996, 2005.

SCHAARS, C.F.; MEINTJES, G.A.; MORRONI, C.; POST, F.A.; MAARTENS, G. Outcome of AIDS-associated cryptococcal meningitis initially treated with $200 \mathrm{mg} /$ day or 400 mg/day of fluconazole. BMC. Infect. Dis., v. 6, p. 118, 2006.

SCHEVEN, M.; SCHWEGLE, F. R. Antagonistic interactions between azoles and amphotericin B with yeasts depend on azole lipophilia for special test conditions in vitro. Antimicrob. Agents. Chemother., v. 38, p. 371-373, 1995.

SHAO, P.L; HUANG, L.M.; HSUEH, P.R. Invasive fungal infection - laboratory diagnosis and antifungal treatment. J. Microbiol. Immunol. Infect., v. 39, p. 178188, 2006.

SHEEHAN, D.J.; HITCHCOCK, C.A.; SIBLEY. C.M. Current and Emerging Azole Antifungal Agents. Clin. Microbiol. Rev., v. 12, p. 40-79, 1999.

SILVA, E.G.; BARONI, F de A.; VIANI, F. C.; RUIZ, L. S.; GANDRA, R.F.; AULER, M. E.; DIAS, A.LT.; GAMBALE, W.; PAULA, C. R. Virulence profile of strains of Cryptococcus neoformans var. grubii evaluated by experimental infection in BALB/C 
mice, and the correlation with exoenzyme activity. J. Med. Microbiol., v. 55, p. 139142, 2006.

SOARES, M.C.B.; PAULA, C.R.; DIAS, A.L.T.; CASEIRO, M.M.; COSTA, S.O.P. Environmental strains of Cryptococcus neoformans variety grubii in the city of Santos, SP, Brazil. Rev. Inst. Med. Trop. S. Paulo, v. 47, p. 31-36, 2005.

SOBEL, J.D. Combination therapy for invasive mycoses: evaluation of past clinical trial designs. Clin. Infect. Dis, Suppl 4, p. S224-227, 2004.

SOUZA, L.K.H.; FERNANDES, O.F.L.; KOBAYASHI, C.C.B.A.; PASSOS, X. S.; COSTA, C.R.; LEMOS, J.A.; SOUZA-JÚNIOR,' A.H.; SILVA, M.R. R. Suscetibilidade antifúngica de isolados clínicos e ambientais de Cryptococcus neoformans na cidade de Goiânia, Goiás, Brasil. Rev. Inst. Med. trop. S. Paulo, v. 47, p. 253-256, 2005.

STEENBERGEN, J.N.; CASADEVALL, A. Prevalence of Cryptococcus neoformans var. neoformans (Serotype D) and Cryptococcus neoformans var. grubii (Serotype A) Isolates in New York City. J. Clin. Microbiol., v. 38, p. 1974-1976, 2000.

SUGAR, A. M. Use of amphotericin B with azole antifungal drugs: what are we doing? Antimicrob. Agents Chemother., v. 39, p. 1907-1912, 1995a.

SUGAR, A. M.; HITCHCOCK, C. A., TROKE, P. F.; PICARD, M. Combination therapy of murine invasive candidiasis with fluconazole and anphotericin $B$. Antimicrob. Agents Chemother, v. 39, p.598-601, 1995b.

TE DORSTHORST, D. T. A.; VERWEIJ ,P. E.; MELETIADIS , J.; M.; BERGERVOET ,N. C. PUNT; MEIS, J. F. G. M.; MOUTON,J. W. In Vitro Interaction of Flucytosine Combined with Amphotericin B or Fluconazole against Thirty-Five Yeast Isolates Determined by both the Fractional Inhibitory Concentration Index and the Response Surface Approach. Antimicrob. Agents Chemother., v.46, p. 2982-2989, 2002.

THIPINE, M.; LETSCHER-BRU, V.; HERBRECHT, R. Amphotericin B and its new formulations: pharmacologic characteristics, clinical efficacy, and tolerability. Transpl. Infect. Dis., v. 1, p. 273-283, 1999.

VAZQUEZ, J.; ARGANOZA, M.; VAISHAMPAYAN, J.; AKINS, R. In vitro interaction between amphotericin $\mathrm{B}$ and azoles in Candida albicans. Antimicrob. Agents Chemother., v. 40, p. 2511-2516, 1996.

VIUDES, A.; PEMAM, J.; CANTON, E.; LOPES-RIBOT., J; GOBERNADO, M. The Activity of Systemic antimycotic drug combinations. Rev. Esp. Quimioter., v. 14, p. 30-39, 2001.

ZARAGOZA, O.; MIHU, C.; CASADEVALL, A.; NOSANCHUK, J.D. Effect of amphotericin $B$ on capsule and cell size in Cryptococcus neoformans during murine infection. Antimicrob. Agents Chemother., v. 49, p. 4358-4361, 2005.

YAMAZUMI, T.; PFALLER, M.A.; MESSER, S.A.; HOUSTON, A.K.; BOYKEN, L.; HOLLIS, R.J.; FURUTA, I.; JONES, R.N. Characterization of Heteroresistance to 
Fluconazole among Clinical Isolates of Cryptococcus neoformans. J. Clin. Microbiol., v. 41, p. 267-272, 2003.

WALSH, T.J.; KASAI, M.; FRANCESCONI, A.; LANDSMAN, D.; CHANOCK, S.J. New evidence that Candida albicans possesses additional ATP-binding cassette MDR-like genes: implications for antifungal azole resistance. J. Med. Vet. Mycol., v. 35, p. 133-137, 1997.

WASAN, K. M.; J. S. CONKLIN. Enhanced amphotericin B nephrotoxicity in intensive care patients with elevated levels of low-density lipoprotein cholesterol. Clin. Infect. Dis. V.24, p. 78-80, 1997.

WEHN, S.M.; HEINZ, E.R. BURGER, P.C.; BOYKO, O.B. Dilated Virchow-Robin spaces in cryptococcal meningitis associated with AIDS: CT and MR findings. J.

Comput. Assist. Tomogr., v. 13, p. 756-762, 1989. 